\title{
Territorios de la Mirada. Inclusión, Educación y Artes con la Comunidad Refugiada en Grecia
}

\section{Territories of the Gaze. Inclusion, Education and the Arts with the Refugee Community in Greece}

\author{
Carlos Escaño ${ }^{1, *}$, Julia Mañero ${ }^{1}$, José María Mesías-Lema ${ }^{2}$ \\ ${ }^{1}$ Universidad de Sevilla, España \\ ${ }^{2}$ Universidade da Coruña, España
}

\section{DESCRIPTORES: \\ Educación artística \\ Diversidad cultural \\ Migración \\ Personas refugiadas \\ Educación para el desarrollo}

\section{RESUMEN:}

Este artículo presenta los resultados de la práctica e investigación pedagógicas llevadas a cabo dentro del proyecto de cooperación internacional "Artes, cultura y educación para el desarrollo. Educación y cultura para el empoderamiento personal y social de la comunidad migrante refugiada en Polykastro, Grecia”. Un proyecto realizado en Grecia, 2019, organizado por la Universidad de Sevilla y Open Cultural Center, una ONG que atiende educativamente a la comunidad migrante refugiada de la zona, incluyendo el campo de refugiados de Nea Kavala. De manera metodológica, las prácticas educativas artísticas llevadas a cabo -bajo el título Territorios de la mirada- participan de la herramienta analítica del Enfoque de Marco Lógico, con el objetivo prioritario de procurar una atención a la comunidad migrante para generar identidad, apertura cultural e inclusión social. Igualmente se proponen los objetivos específicos de corroborar y ofrecer posibilidades para elaborar un plan de trabajo educativo inclusivo en el desarrollo posterior del proyecto completo. Se han implementado estrategias de investigación basadas en las artes, logrando una mejor imbricación comunitaria y fortaleciendo la dimensión artístico-cultural, lo cual ha beneficiado a los procesos educativos de la persona migrante refugiada y su comunidad, favoreciendo su inclusión y desarrollo.

\section{KEYWORDS:}

Arts education

Cultural diversity

Migration

Refugees

Education for development

\section{ABSTRACT:}

This paper presents the results of the pedagogical praxis and research carried out within the international cooperation project entitled "Arts, culture and education for development. Education and culture for the personal and social empowerment of the migrant refugee community in Polykastro, Greece". This project was developed in Greece, 2019, and organised by the University of Seville and Open Cultural Center, an NGO that provides educational services to the migrant refugee community in the area, including the Nea Kavala refugee camp. The methodological considerations addressed in the art educational practices carried out -entitled Territories of the gaze- are based on the analytical tool of the Logical Framework Approach. This was related to our main objective: providing attention to the migrant community in order to generate identity, cultural openness and social inclusion. The specific objectives are also proposed to corroborate and offer possibilities to elaborate an inclusive educational work plan in the further development of the whole project. Research strategies based on the arts have been implemented, in order to achieve a better community integration and strengthen the artistic-cultural dimension, which has benefited the educational processes of the migrant refugees and their community, and at the same time has favoured their inclusion and development.

CÓMO CITAR:

Escaño, C., Mañero, J. y Mesías-Lema, J. M. (2021). Territorios de la mirada. Inclusión, educación y acciones artísticas con la comunidad refugiada en Grecia. Revista Internacional de Educación para la Justicia Social, 10(2),59-74.

https://doi.org/10.15366/riejs2021.10.2.004

${ }^{*}$ Contacto: jcescano@us.es

ISSN: 2254-3139

revistas.uam.es/riejs 


\section{Introducción}

El presente artículo muestra los resultados de las actividades de investigación y acción pedagógicas llevadas a cabo dentro del proyecto de cooperación "Artes, cultura y educación para el desarrollo. Educación y cultura para el empoderamiento personal y social de la comunidad migrante refugiada en Polykastro, Grecia”. Un proyecto de cooperación realizado en 2019 e implementado por la Universidad de Sevilla en colaboración con la ONG Open Cultural Center (OCC) que trabaja en Polykastro (Grecia), atendiendo desde el punto de vista educativo a la comunidad migrante refugiada de la zona, incluyendo el campo de refugiados de Nea Kavala, sitio a pocos kilómetros de la localidad. El proyecto se centra en fortalecer la dimensión artístico-cultural de la comunidad, en beneficio del proceso educativo de la persona migrante refugiada para su empoderamiento personal y también colectivo, favoreciendo su inclusión social y desarrollo. De esta manera, el proyecto participa de la tradición conceptual y ética relacionada con el enfoque educativo de reconstrucción social, donde el eje central está ubicado en la disipación de estructuras sociales injustas y la promoción de la diversidad social y cultural (Sleeter y Grant, 1988).

El proyecto de cooperación se ha desarrollado como fase de identificación, preceptiva en el inicio de un ejercicio completo de cooperación al desarrollo, y según la metodología que implica el planteamiento del enfoque de marco lógico. Dentro de esta estrategia metodológica se ha valorado como tarea fundamental la implementación de acciones pedagógicas (denominadas Territorios de la mirada) y que han contribuido de manera decisiva al recorrido investigador pertinente que debe mantener un proyecto de este tipo. Prácticas docentes que han servido para revelar posibilidades y conclusiones, las cuales han repercutido en la tarea inclusiva de empoderamiento tanto individual como colectivo de la comunidad migrante. La importancia de esa práctica pedagógica como mecanismo metodológico se ha mostrado esencial y ha ofrecido indicios pertinentes para el diseño de todo un plan de acción educativa, un resultado específico del conjunto del proyecto para su desarrollo a posteriori.

Finalmente, cabe señalar, que el proyecto de cooperación "Artes, cultura y educación para el desarrollo" mantiene unos antecedentes que parten del análisis del contexto ya efectuado en diciembre de 2017, momento en el que se toma contacto con OCC, se visita la zona y se establecen las bases de colaboración posterior. Tras la preparación del proyecto, se solicita la financiación universitaria pertinente en el año 2018 y posteriormente se ha podido llevar a cabo desde finales de septiembre y el mes de octubre de 2019. Todo proyecto de cooperación en un territorio está condicionado por ese análisis previo del contexto, el cual también sirvió para contactar con la Universidad Aristóteles

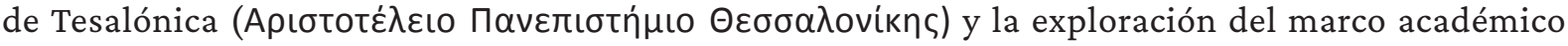
institucional. El coordinador del presente proyecto participó como parte del comité científico del Seminario Internacional "Research and praxis for social engaged arts education" de la Sociedad Internacional de Educación a través del Arte - International Society of Education through Art (InSEA), celebrado en julio de 2018 en la Universidad Aristóteles, en Tesalónica, capital de la región de Macedonia Central, y ciudad de referencia cercana al contexto migrante de Polykastro. 
Figura 1

Conjunto de fotogramas del inicio del trabajo audiovisual Territorios de la mirada (Territories of the gaze) (2019), del colectivo Communiars ${ }^{1}$
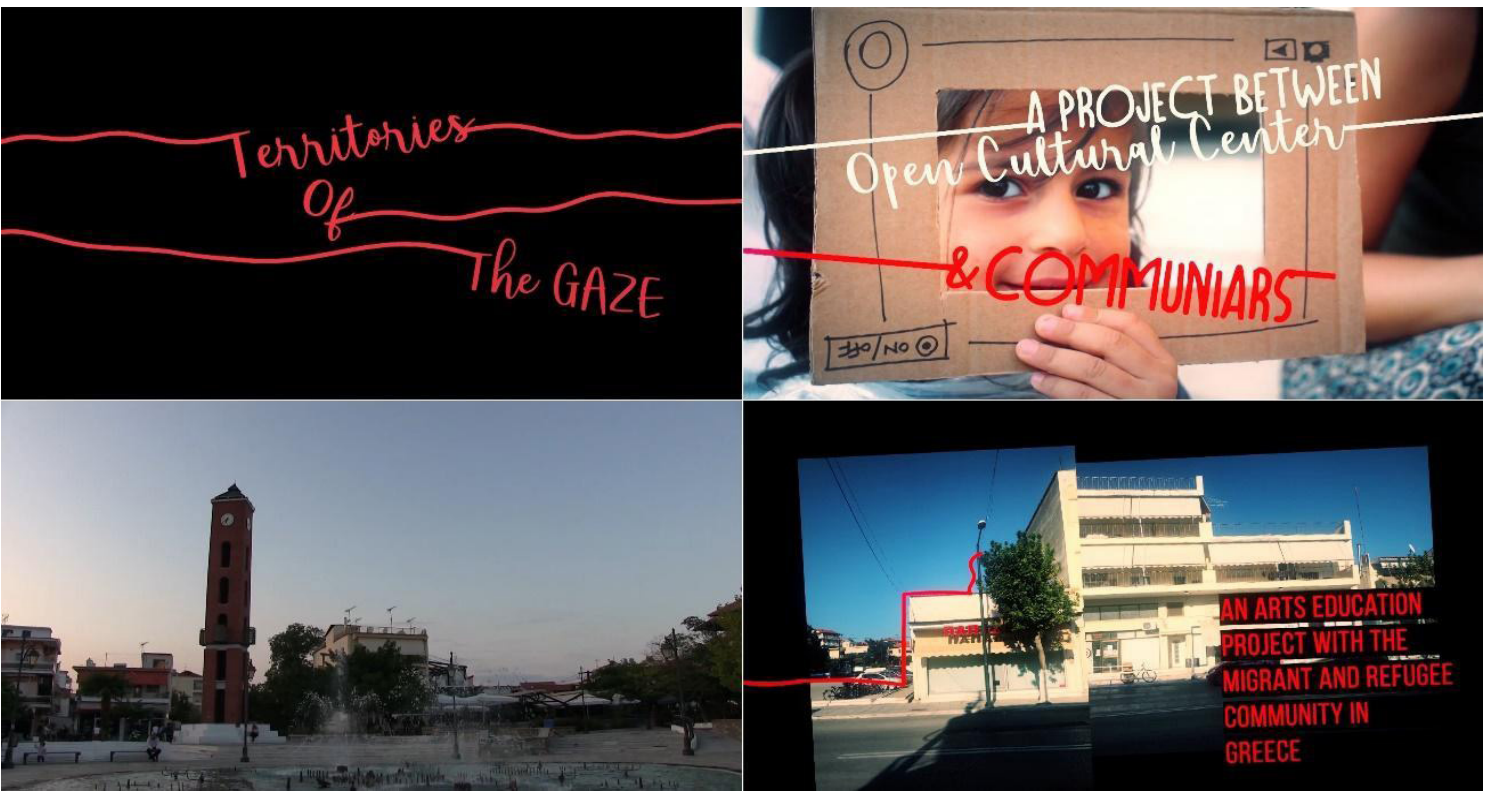

\section{Revisión de la literatura}

\subsection{Educación, refugio y migración en Europa: El contexto griego}

En Europa, desde la explosión migratoria del 2015, la petición de asilo es una constante con tintes dramáticos. Desde 2015 más de un millón de personas llegaron vía marítima de manera precaria y la gran mayoría procedían de Siria, Irak y Afganistán. Se presentaron 1,5 millones de solicitudes de asilo en los principales países europeos de acogida, Alemania y Suecia, y las llegadas a Chipre, Malta, Italia, España y Grecia continuaron en los siguientes años, aunque ahora el límite de solicitudes se ha establecido en 200.000 entre 2017 y 2019 (Oficina del Alto Comisionado de las Naciones Unidas para los Refugiados [ACNUR], 2020). Cientos de miles de personas permanecen en una inquietante situación de espera y lentitud burocrática, conviviendo en espacios poco preparados para la dignidad de las personas y menos en una época azotada por la pandemia vírica del Covid-19. Un ejemplo muy significativo y simbólico de esta situación fue el terrible incidente de agosto de 2020, cuando el campo de refugiados de Moria, situado en Mitilene, Lesbos (Grecia), fue arrasado por un incendio. Un campo con capacidad limitada para una ya difícil convivencia de 3.000 personas, pero que estaba sobresaturado con 13.000 personas. Un escenario dramático que ejemplifica claramente la imposibilidad de un mantenimiento de medidas de seguridad de salud en nuestro momento actual y que refleja la alarmante falta de dignidad con la que se vive en este tipo de situaciones.

La política de asilo de la Unión Europea (UE) lleva tiempo buscando establecer un territorio común de protección y solidaridad que se base en un procedimiento de estatuto y asilo comunes, tal y como se afirma en la investigación desarrollada para la Comisión de Libertades Civiles, Justicia y Asuntos de Interior del Parlamento Europeo y encargado, supervisado y publicado por el Departamento de Política de Derechos de los Ciudadanos y Asuntos Constitucionales (Wagner et al., 2016). La UE ha evidenciado que su normativa común de asilo es insuficiente y los resultados de la política de cada país miembro son muy

1 Las imágenes reseñadas para la ilustración de la praxis educativa experimental en el presente artículo son fotogramas que proceden del documento audiovisual “Territorios de la mirada” (Territories of the gaze) (2019), un trabajo que recoge esa práctica pedagógica y artística realizada. Un documento bajo licencia Creative Commons BY-NC-SA Por este motivo legal, se tiene el permiso explícito para utilizar, difundir y compartir sus imágenes en este texto. 
desiguales, derivando en múltiples enfoques e interpretaciones desde las esferas mediáticas y políticas (Triandafyllidou, 2018). Las estrategias básicas migratorias de la UE se corresponden con el enfoque de estratificación cívica propio de la implementación política de los programas de bienestar para migrantes que se han propuesto, provocando una descoordinación y desnivel de acción institucional: tal como señala Lydia Morris y recoge Rosenberger (2019), la fragmentación, y su lógica inestable, es una estrategia política para gobernar la migración irregular y el bienestar en los países europeos, la cual permite a los gobiernos conceder distintas categorías administrativas de migrantes. En consecuencia, también se otorgan derechos sociales inferiores a los que disfrutan los nativos, repercutiendo directamente en derechos tan fundamentales como el acceso a la sanidad y a la educación. Ante esta situación fragmentaria los diferentes estados europeos acometen la organización del flujo migratorio de diferente manera y el caso del gobierno griego supone un caso muy significativo por su relevancia geopolítica estratégica y la magnitud del volumen migratorio: los últimos datos arrojan cifras de 80.468 personas totales refugiadas o en situación similar a las refugiadas, 105.698 pendientes de asilo (ACNUR, 2020).

Figura 2

Campo de refugiados de Nea Kavala. Conjunto de fotogramas de Territorios de la mirada (Territories of the gaze) (2019), del colectivo Communiars

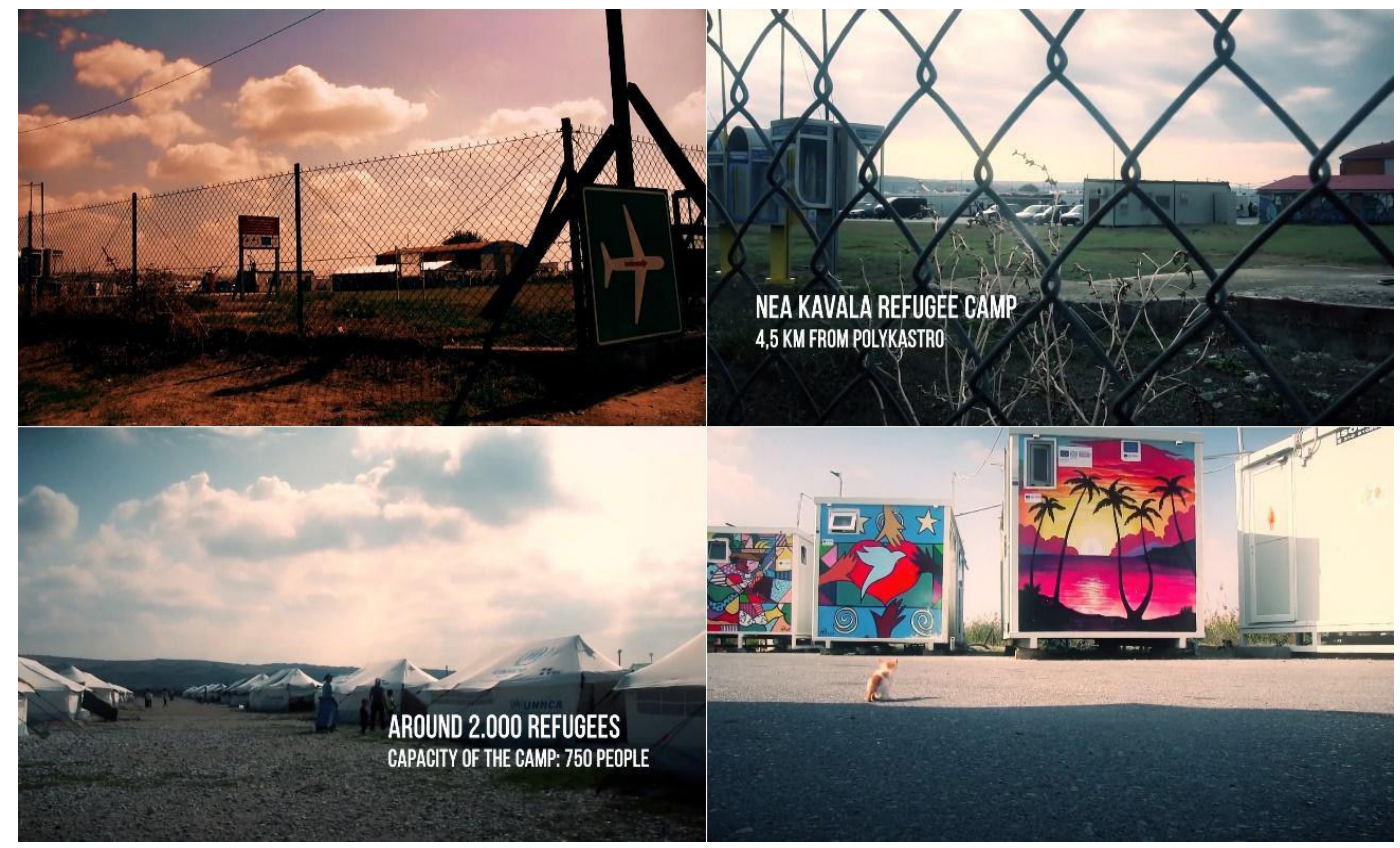

En esta situación la atención educativa se ha convertido en una prioridad social para el ejercicio de inclusión y normalización política no solo de Grecia, sino para el conjunto continental europeo. Más allá de la respuesta política institucional helena, ha sido el propio profesorado, como cuerpo sujeto a un código deontológico, el que denuncia que una exclusión social a la comunidad migrante entraría en conflicto con su responsabilidad respecto a los educandos (Sarah y Delvino, 2019). El sector griego de educación está afrontando los problemas de inclusión de niños refugiados en las escuelas griegas y para ello se necesita la participación de la sociedad civil con su apoyo en la promoción y la sensibilización, de cara a la mejora de una educación intercultural y la cohesión social entre todo el estudiantado, autóctono y migrante. En este ejercicio de soporte y gestión educativas, diversas corporaciones no gubernamentales han favorecido una oferta pedagógica no formal en Grecia focalizándose en habilidades comunicativas idiomáticas, sociales y apoyo psicosocial vinculado a todo desarrollo de los procesos de enseñanza y el aprendizaje (UNICEF, 2017). Dentro de este espacio de intervención político-cultural y aprendizaje se enmarca la acción social de la ONG Open Cultural Center. Una asociación que facilita herramientas a los migrantes para acceder a oportunidades y construir un futuro y presente, mientras promueve la igualdad y la inclusión dentro de las comunidades locales a través del intercambio intercultural y la educación (OCC, 2021). 
OCC es una ONG de Barcelona, España, que trabaja en el norte de Grecia, desde hace unos años en la localidad de Polykastro y municipios aledaños como Axiópolis. Contextos cercanos al campo de refugiados de Nea Kavala (a 4,5 km de Polykastro), el cual tiene una cabida para 750 personas, pero que ha llegado a estar a más del doble de su capacidad en momentos álgidos, incluso en época de confinamiento por Covid-19. La comunidad migrante que reside en la zona ha llegado a sumar más de trescientas personas. OCC se esfuerza por convertirse en un actor clave dentro de la red comunitaria de organizaciones que trabajan de manera colaborativa para la mejora de la inclusión social y económica de los migrantes a nivel local, procurando promocionar políticas que protejan los derechos de los migrantes y favorezcan su inclusión en una sociedad europea diversa (OCC, 2021). OCC se fundó en abril de 2016 como iniciativa colectiva de voluntarios independientes dentro del campo de refugiados de Idomeni (Grecia). Con el tiempo y el desarrollo de la ONG, su evolución ha generado un sello de identidad fundamental en el proyecto: OCC se nutre tanto de un voluntariado internacional venido de diferentes partes del mundo, como de voluntariado residente, es decir, personas migrantes de la propia comunidad refugiada que conviven en Polykastro, sea en la misma ciudad o en Nea Kavala. La población migrante mayoritaria en estos años proviene de Oriente medio (Siria, Irak y Afganistán) y la actividad pedagógica de OCC de la que se benefician fundamentalmente se visibiliza a partir dos de sus proyectos educativos principales:

- Proyecto de clases de idiomas. Actualmente, el inglés se configura como oferta lingüística clave para la comunidad migrante. Una propuesta pedagógica necesaria para el desarrollo de habilidades sociales que está orientada para todas las edades.

- Proyecto Kindergarten. Un proyecto dedicado a la educación infantil y que está desarrollado junto con la organización sin ánimo de lucro Refugee Trauma Initiative (RTI). Este proyecto va destinado a niños de 3 a 5 años.

Figura 3

Open Cultural Center. Conjunto de fotogramas de Territorios de la mirada (Territories of the gaze) (2019), del colectivo Communiars

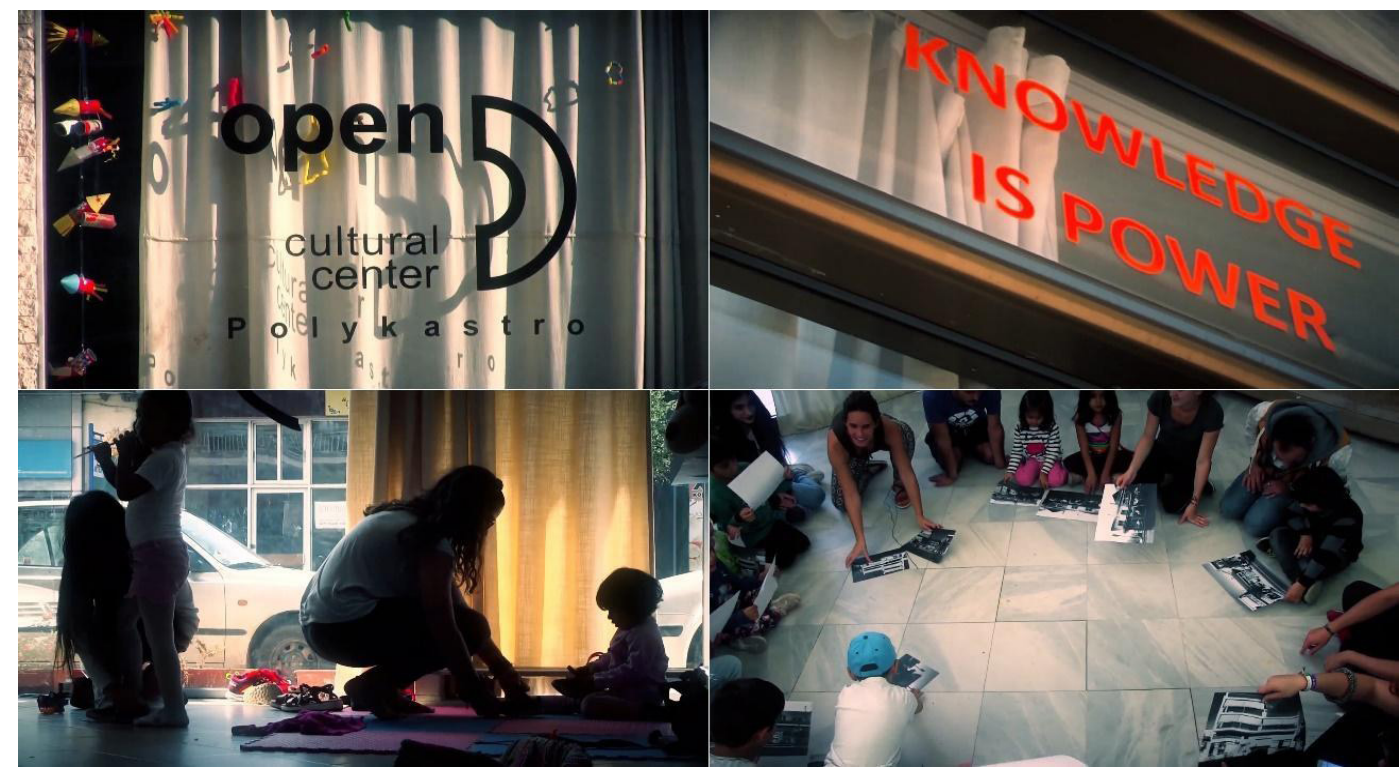

\subsection{Educación artística, diversidad cultural y migración}

La práctica artística y cultural no está catalogada como área de prioridad en ejercicios críticos de emergencia dentro de la ayuda humanitaria (Carrillo-Jaimes y Arcos-González, 2004). Pero es oportuno reseñar que cuando se desencadena una situación catastrófica no sólo afecta a las dimensiones físicas y materiales de una comunidad, sino también a las conexiones sociales y emocionales que unen a las personas (Hirsu et al., 2020). En un proceso migratorio forzoso de gran volumen dentro de un contexto determinado y que se perpetúa en el tiempo, la tarea educativa y artística cobra una relevancia social clara: las 
producciones culturales proporcionan una enorme capacidad para transformar percepciones, actitudes y sistemas de creencias integrales (Anttila y Suominen, 2019). Las profesoras Eeva Anttila y Anniina Souminen (2019) destacan el valor de las artes para considerarse tanto una necesidad existencial de los seres humanos como un canal para dar sentido a las experiencias y ejercer el cambio social:

Art is an expression of social imagination, a tool for building equitable cultural commons, and a means by which hope can be nurtured. Arts education that is sensitive and responsive to cultural diversity, is holistically reflective, critical ofits self and society, and aims at building commons through ethical and deeply caring practices has potential to transform individuals and institutions ${ }^{2}$. (Anttila y Suominen, 2019, p. 5)

Esta base socioeducativa de la práctica artística justifica su participación activa en contextos donde existe una presencia migratoria importante: contextos donde el proceso de construcción cultural es inevitablemente diverso. Como recoge Chalmers (2003), a partir de lo observado por la Association for Supervision and Curriculum Development (1992), las artes son clave para la comprensión de cada cultura y, en consecuencia, un elemento de gran relevancia sería el desarrollo de su estudio y producción dentro de entornos educativos. Aún más reseñable es su estudio si consideramos que aquellos espacios donde la migración juega un papel esencial en la construcción de los lazos comunitarios, la diversidad cultural implica un ejercicio sensible y determinante. Se hace más necesario entonces fomentar una comprensión del arte desde las diferentes perspectivas de las diversas culturas dentro de un espacio común, para realzar su comprensión y demostrar a los educandos que el arte constituye una parte importante de las actividades humanas y el cambio social.

Estos fundamentos básicos suponen las bases para las acciones pedagógicas llevadas a cabo en el proyecto de cooperación internacional "Artes, Cultura y Educación para el Desarrollo", las cuales funcionan como mecanismo de investigación docente. El proyecto se adscribe a la voluntad por garantizar el derecho humano a la cultura para todos, con un enfoque profundo que llegue a las comunidades más vulnerables (Hirsu et al., 2020), con la firme intención del desarrollo de una perspectiva de la educación artística que triangule entre la solidaridad, la esperanza y la justicia social. Desde nuestra experiencia en el trabajo educativo y artístico de cooperación se nos ha revelado, a lo largo de los últimos años, que existen grandes posibilidades y potencialidades en la dimensión artística por y para el cambio social: nuestro espacio web www.communiars.org recoge las dinámicas y ejemplos de proyectos como "Educación Artística y Desarrollo Humano”, llevado a cabo en India, durante los años 2015 a 2018, junto a la fundación Vicente Ferrer, y que nos sirve como antecedente y espacio de aprendizaje educativo y vital (Communiars, 2021). La educación artística en un terreno de cooperación no únicamente fomenta la colaboración y la diversidad, sino una serie de valores transversales a través de un aprendizaje reflexivo y crítico, donde se destacan el desarrollo de la igualdad, el estímulo de la empatía y autoestima, y el fomento de la esperanza (Escaño, 2019).

De esta manera, tal y como señala Saura (2015), la educación para la justicia social mantiene como objetivo prioritario promover la participación social y, en esta línea de trabajo, considera al arte como un medio a través del cual este objetivo se puede conseguir. Siguiendo este planteamiento, la profesora e investigadora Dipti Desai (2017) reivindica el papel activista de las artes como una pedagogía de la posibilidad. La educadora artística defiende el espacio que brindan las artes hacia la experimentación, la imaginación y la visualización de alternativas posibles. Este enfoque orientado hacia la reconstrucción social subyace no solo en el proyecto de cooperación reseñado, sino que conecta con la mirada educativa y cultural de OCC y su labor de inclusión social.

2 Traducción de los autores: "El arte es una expresión de la imaginación social, una herramienta para construir un patrimonio cultural equitativo y un medio para alimentar la esperanza. La educación artística, que es sensible y receptiva a la diversidad cultural, es holísticamente reflexiva, crítica consigo misma y con la sociedad, y tiene como objetivo la construcción de bienes comunes a través de prácticas éticas y profundamente solidarias, tiene el potencial de transformar a los individuos y a las instituciones”. 


\section{Método}

Las dinámicas artísticas docentes llevadas a cabo se circunscriben dentro de un proceso metodológico de investigación propio de la fase de identificación en un proyecto de cooperación. El conjunto global de un proyecto de cooperación al desarrollo mantiene un primer proceso preceptivo, la denominada fase de identificación. Fase que se traduce en un ejercicio de investigación que favorece la diagnosis para la delimitación y definición del espacio, tiempo y métodos de actuación en el desarrollo a posteriori del proyecto. Una pieza fundamental dentro de un proyecto más amplio que depende de esa identificación. Lo que se deduzca del ejercicio de investigación inicial de identificación servirá para diseñar el plan de acción futuro y venidero en un proyecto más amplio. Para la realización de la tarea de identificación se acude a la herramienta metodológica del enfoque del marco lógico (EML), cuyo objetivo es facilitar el proceso de conceptualización, diseño, ejecución y evaluación del proyecto (Ortegón et al., 2015). El EML mantiene cuatro niveles analíticos: análisis de involucrados, análisis de problemas, análisis de objetivos y análisis de alternativas. Tras los diferentes tipos de análisis efectuados, donde se estudian los distintos espacios educativos de OCC, se advierte que su proyecto Kindergarten es el más adecuado para centrarse en la planificación futura de un proyecto de cooperación educativa más amplio. El proyecto de Kindergarten, como señalábamos previamente, está coorganizado junto a Refugee Trauma Initiative (RTI) y orientado a niñas y niños entre 3 y 5 años de edad, es un servicio gratuito ofrecido por OCC, lo que permite a las familias migrantes brindarles un lugar seguro a sus hijos para jugar y aprender, favoreciéndoles igualmente a las familias la autonomía pertinente para trabajar y/o conciliar con otras facetas sociales que puedan desarrollar. El proyecto funciona desde la fundación de la institución y es uno de los más estables en lo referente al voluntariado residente implicado. Por este motivo es el proyecto seleccionado para realizar una práctica pedagógica experimental que corrobore y posibilite más pistas educativas en el diseño de ese plan a largo plazo. Como parte fundamental dentro del EML desarrollado ha sido básico la implementación de esta intervención pedagógica experimental. Su importancia requiere del desarrollo descriptivo y analítico para este artículo. La intervención pedagógica experimental ha supuesto un ejercicio metodológico de investigación docente en sí, fundamentado principalmente en un método de observación participante completa a partir de instrumentos y una perspectiva relacionada con la investigación basada en las artes, la cual mantiene tradición en el uso de tales procedimientos metodológicos interactivos (Agra, 2005; Eisner y Barone, 2012; Marín Viadel, 2005, 2011). Es decir, la generación de una estrategia educativa como la que se expone ha servido como espacio de investigación del que, por su naturaleza favorecedora de aprendizaje y descubrimiento informativo, nos beneficiamos de cara al desarrollo del proyecto: el trabajo de intervención pedagógica persigue esencialmente los objetivos de evidenciar desde la práctica las posibilidades reales para poder implementar un plan completo pedagógico en una fase siguiente del proyecto y, asimismo, corroborar aquellos aspectos estudiados en la fase de análisis de objetivos y de alternativas del estudio EML.

La acción experimental de observación participante completa permite a los miembros coordinadores e investigadores de la acción convertirse en parte del grupo y comunidad estudiada, participando de las actividades diarias, las interacciones y los acontecimientos del grupo de personas como uno de los medios para aprender los aspectos explícitos y tácitos de sus rutinas de vida y su cultura (DeWalt y DeWalt, 2011). La observación participante ofrece una perspectiva holística, al observar y participar al mismo tiempo de un fenómeno desde su máxima cotidianeidad. Se parte así de un interaccionismo simbólico en el que, para comprender la realidad, los investigadores necesitan interaccionar, compartir y comprometerse con la realidad que investiga (Massot-Lafon et al., 2009). Así, dentro de este enfoque, se propone una experiencia educativa en la que el arte es el eje central del trabajo pedagógico. La muestra de la que se parte es de 40 niños y niñas de origen sirio. La Kindergarten en 2019 cuenta con 20 usuarios menores de 5 años (OCC, 2020) manteniendo una aproximada paridad de sexo; en las actividades propuestas también han intervenido otros 20 niños más, de edades que oscilan entre los 6 y 8 años (12 niñas y 8 niños), que forman parte de un gran grupo de usuarios de OCC, conjunto que asciende a un total de 70 niños/as divididos en tres grupos de edades como alumnado de los cursos de idiomas de OCC (2020). Para la realización de la intervención pedagógica contamos con la implicación docente de un grupo de 7 monitores voluntarios residentes y voluntarios internacionales.

Como señalan Massot-Lafon y otros (2009), la planificación en la observación participante educativa tiene un carácter inductivo, emergente y flexible. Las autoras, basándose en la tradición metodológica cuali- 
tativa recogida por Ruiz-Olabuénaga (1999), resaltan igualmente la gran importancia de establecer relaciones de confianza y seguridad con los informantes para que se expresen con total libertad y se muestren sin reticencias, facilitando así las indicaciones para seleccionar la estrategia educativa más adecuada:

- Acomodación en las rutinas y modos de hacer de los informadores, en este caso, el alumnado.

- Soslayar la parafernalia social del investigador científico.

- Evitar comentarios evaluativos de las personas.

- Mostrar interés por toda información, aunque pudiera parecer irrelevante.

A partir de estos principios metodológicos, lo verdaderamente relevante es centrarse en el proceso por encima de la consecución de un mejor resultado técnico procedimental. Las dinámicas metodológicas se realizan en colectivo dando prioridad a la participación, al diálogo, al carácter lúdico y a la apertura del alumnado hacia la comunidad.

Así, se desarrolla una serie de acciones educativo-artísticas experimentales a lo largo de diferentes sesiones y que mantiene las siguientes características metodológicas recogidas en el Cuadro 1.

Cuadro 1

Objetivos y estructura metodológica

Título Territorios de la mirada

Objetivo general: existe un objetivo general en relación con la proyección educativa. Es decir, un objetivo que abraza no solo la tarea de esta práctica pedagógica experimental, sino que orienta el camino de todo el proyecto y sentido pedagógico del trabajo de cooperación al desarrollo que se propone. Este objetivo se centra en procurar la atención y aproximación al entorno de la comunidad para generar identidad, apertura cultural democrática e inclusión social a través de estrategias visuales.

Objetivos Objetivos específicos para con el proyecto: como hemos señalado anteriormente, existen asimismo objetivos específicos orientados a una tarea de verificación y validación de resultados y posibilidades:

Por un lado, se trata de corroborar aquellos aspectos estudiados en la fase de análisis de objetivos y de alternativas del estudio EML.

Por otro lado, se pretende comprobar desde la praxis educativa las posibilidades reales que ofrece la práctica educativa artística para tenerlas en cuenta como parte de un ulterior plan completo de acción pedagógica. Comprobar así cómo funciona un planteamiento educativo y artístico dentro de las lógicas del reconstruccionismo social dentro de la comunidad migrante refugiada.

Fase 1. Trazando nuestro territorio. Acercamiento visual y gráfico al entorno urbano y social. Procedimiento de intervención gráfica a través del dibujo en fotografías de gran dimensión. Ejercicio colectivo.

Fase 2. Visibilizar la calle. Vivencia del barrio desde un punto de vista artístico. Procedimiento de intervención con pintura acrílica en el exterior y con formatos medios. Ejercicio individual.

Estructura metodológica Fase 3. Toma la plaza. Experimentación artística colectiva en uno de los espacios urbanos más icónicos del municipio. Procedimiento de intervención con pintura acrílica con un gran formato y en un entorno social fuera de las instalaciones de OCC. Ejercicio colectivo.

Fase 4. Habitar la mirada. Experimentación digital artística dentro de un escenario de chroma key en tiempo real y a partir de los trabajos gráficos realizados previamente. Ejercicio colectivo.

Finalmente, en las instalaciones de OCC, se preparó una exhibición pública, una proyección del trabajo audiovisual "Territorios de la mirada" (Territories of the gaze), documento que recoge la acción educativa realizada. Posteriormente, se mantuvo el correspondiente diálogo con la comunidad participante. 


\section{Resultados}

Los resultados obtenidos de la intervención pedagógica desarrollada pueden observarse de manera íntegra en el trabajo audiovisual reseñado: "Territorios de la mirada (Territories of the gaze)" https://communiars. org/2019/11/11/territorios-de-la-mirada-territories-of-the-gaze/.

Para la consecución de resultados se propone un desarrollo de las acciones educativas y artísticas que han sido articuladas a partir de criterios establecidos gracias al estudio previo realizado. Como se ha hecho explícito anteriormente, estamos en una fase de identificación para la definición del contexto y métodos de actuación en el desarrollo a posteriori del proyecto y, de manera coherente, las actividades que se implementan deben favorecer esa identificación y diagnosis. Los criterios de valoración, por tanto, se ponen en relación con los cuatro niveles analíticos que mantiene el EML: análisis de involucrados, de problemas, de objetivos y de alternativas. De los cuales se deduce, tal y como se recoge en Escaño y otros (2021), que los tres principales criterios de valoración de las actividades se organizan en torno a:

Por un lado, la necesidad de potenciación de inclusión de las familias migrantes en la comunidad griega; por otro lado, una mejor integración institucional y posibilidad de nexos con las escuelas; $y$, por último, que se favorezca la posibilidad de promoción de una colaboración universitaria internacional y nacional.

A continuación, y bajo este marco de acción y criterios, se pensaron actividades de las que detallamos sus resultados en las siguientes fases, tanto textual como visualmente:

1. Trazando nuestro territorio. Dentro de las propias instalaciones de la ONG Open Cultural Center (OCC) comienza la primera fase de esta intervención pedagógica. El grupo de voluntarios internacionales y residentes en colaboración con los tres integrantes del proyecto de Cooperación de "Artes, Cultura y Educación para el Desarrollo", coordinan una primera acción educativa y artística centrada en la representación gráfica: con el grupo de niños y niñas migrantes se realiza un acercamiento al contexto urbano y social. En un papel continuo de gran formato de $100 \mathrm{~cm}$ x $300 \mathrm{~cm}$ se fijan imágenes fotográficas en blanco y negro que representan la calle donde están situadas las instalaciones de OCC, la arteria principal de la localidad de Polykastro. Entre todos los niños participantes y los monitores educativos, de manera colectiva, se intervienen las fotografías rotulando con tinta roja y delimitando el contorno de las diferentes imágenes de la calle y edificios representados. La práctica está planteada para interiorizar el alrededor cotidiano a través de la observación y atención focalizada en esos detalles. El formato monocromático de las fotografías y el trazo en diferente color resalta la delimitación de los detalles. Con esta acción cada niño y niña se introduce en el concepto de representación gráfica a partir de una guía visual que, además de facilitar la psicomotricidad fina en niños y niñas de edades tempranas, les permite a su vez reflexionar desde lo visual sobre la toma de conciencia de su alrededor inmediato.

\section{Figura 4}

Trazando nuestro territorio. Conjunto de fotogramas de Territorios de la mirada (Territories of the gaze) (2019), del colectivo Communiars

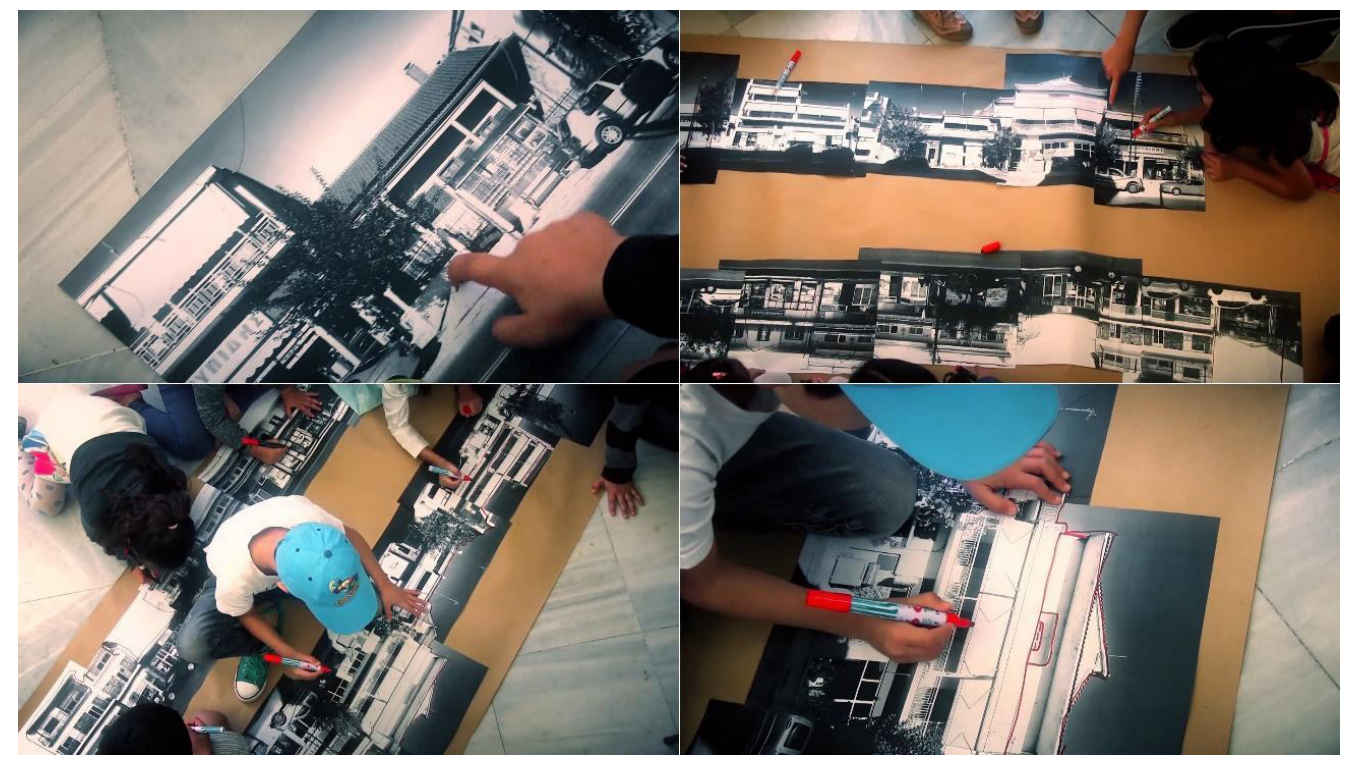


2. Visibilizar la calle. Esa introducción a la acción gráfica de la primera fase, realizada dentro de las instalaciones de OCC, suponía un preámbulo de doble vía, tanto gráfica como social: una aproximación al manejo del trazo y las posibilidades gráficas y, por otro lado, una introducción a la toma de conciencia del alrededor social. Tras esta primera práctica pedagógica, damos paso a una segunda fase que se realiza en las inmediaciones de OCC y se caracteriza por la apertura al exterior y la expansión de las fronteras del espacio educativo, aprovechando las ventajas y eficacia en el aprendizaje, destacando esencialmente la mejora de las competencias sociales y comunicativas de los niños (Robertson, 2014). Se trata de realizar un recorrido de la calle a partir de la mirada subjetiva. Para ello se realizaron visores manufacturados con un diseño sencillo sobre cartón reciclado; estos visores son utilizados por los niños y niñas para encuadrar su mirada, seleccionando el plano que prefieran. Se trata de identificar las fotografías anteriormente intervenidas de manera gráfica con los contornos delimitados en rojo y, de esta manera, se ayuda a la reflexividad a través de sus miradas. Posteriormente, una vez encuadrado y seleccionado el plano, representan lo que están visualizando a través de una práctica pictórica en un formato individualizado.

Figura 5

Visibilizar la calle. Conjunto de fotogramas de Territorios de la mirada (Territories of the gaze) (2019), del colectivo Communiars

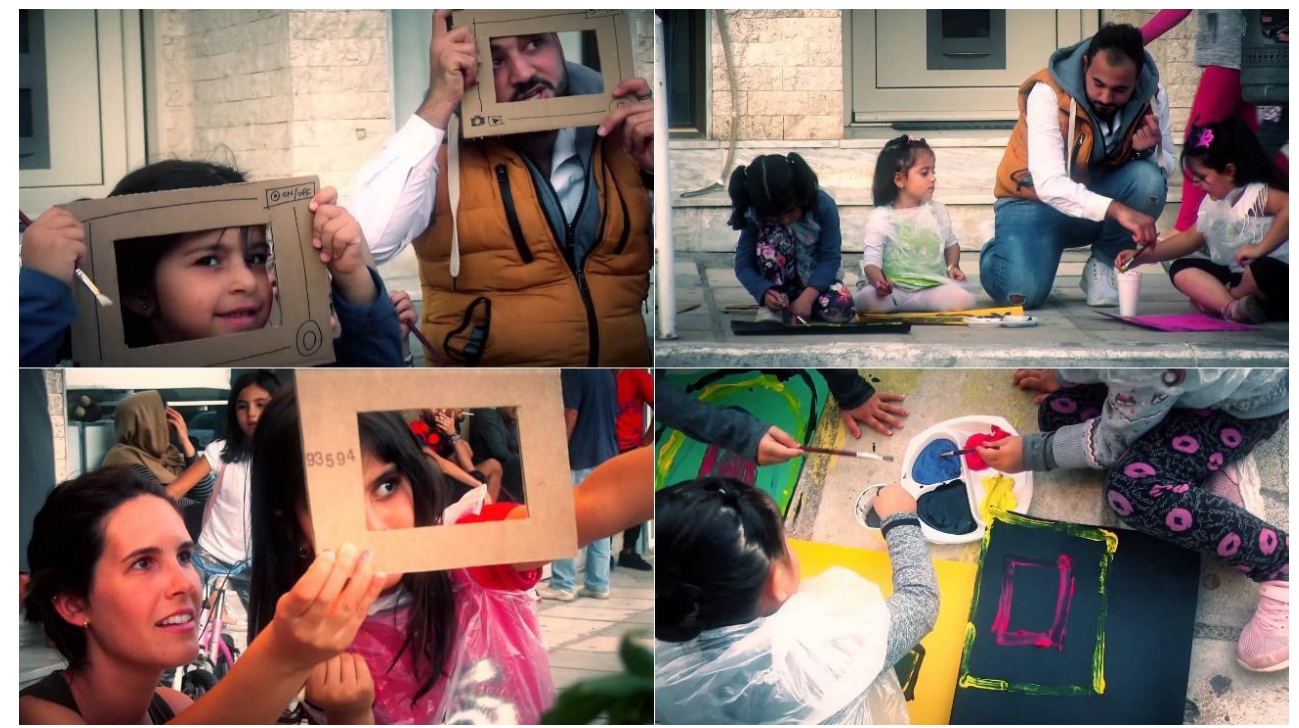

Figura 6

Obras pictóricas realizadas por los niños y niñas de OCC durante la fase Visibilizar la calle. Conjunto de fotogramas de Territorios de la mirada (Territories of the gaze) (2019), del colectivo Communiars

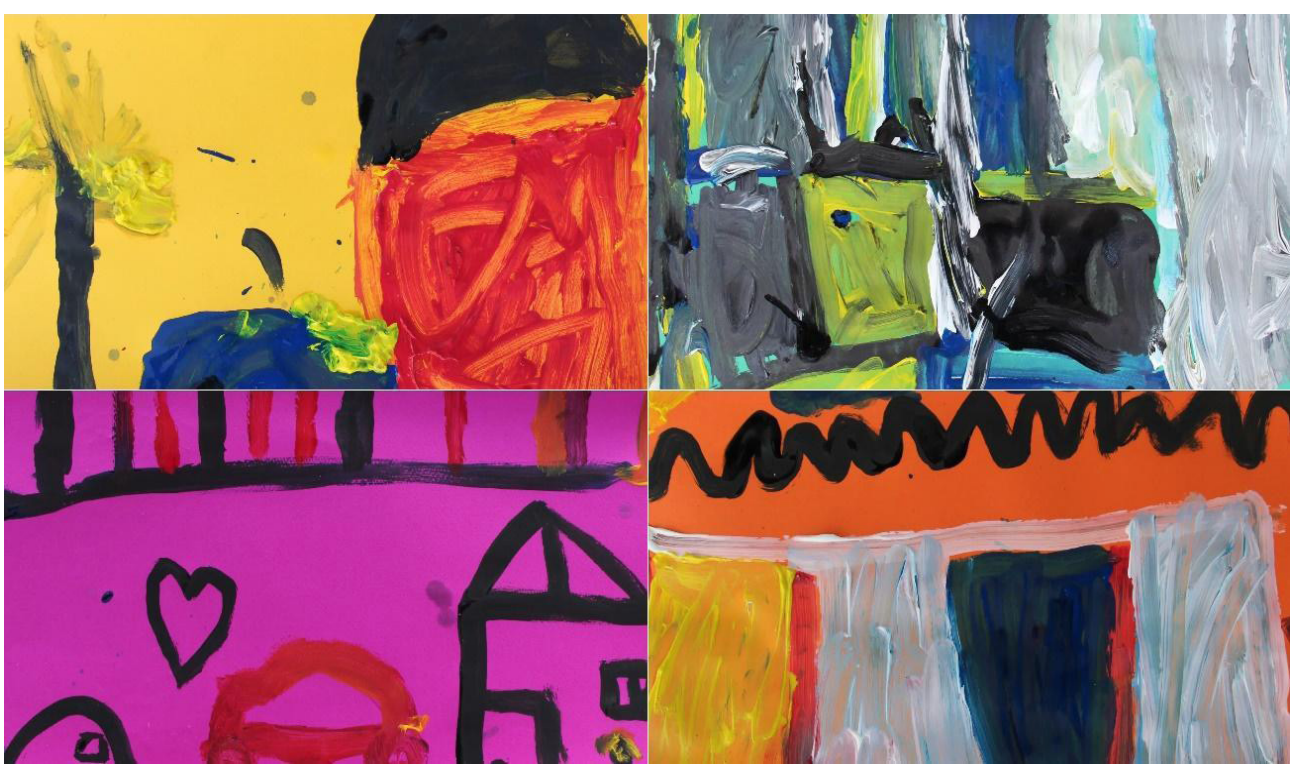


3. Toma la plaza. Como tercer paso se realiza una intervención colectiva y pictórica buscando desde sus aspectos formales, metodológicos y de contenidos, la toma de conciencia de la apertura social, la diversidad y la inclusión comunitaria. Tras el ejercicio de reflexión visual particular de la fase 2, donde la mirada ha sido focalizada en la representación del entorno urbanístico y social más inmediato de OCC, se sigue explorando el exterior y entorno social en las dinámicas de la presente fase, pero esta vez se realiza en colectivo, apelando de manera simbólica a la acción en el entorno comunitario, no solo como individuos sino como grupo. De nuevo se utiliza un gran formato: dos pliegos de papel continuo blanco de $100 \mathrm{~cm} \mathrm{x}$ $400 \mathrm{~cm}$ cada uno para realizar un trabajo pictórico de manera grupal donde todos -voluntariado internacional y residente, niños y niñas-intervienen. La acción se realiza en uno de los entornos más representativos de la localidad: la gran plaza del municipio, punto neurálgico local de actividad social y comercial. La pretensión se centra en generar lazos de comunidad e inclusión entre la población migrante para con el contexto griego y viceversa. Al margen del resultado técnico, el proceso de trabajo fue realizado con una actitud lúdica e interactiva permitiendo un aprendizaje alejado de la mera instrucción y focalizado en la tarea inclusiva a través de la expresión artística.

\section{Figura 7}

Toma la plaza. Conjunto de fotogramas de Territorios de la mirada (Territories of the gaze) (2019), del colectivo Communiars

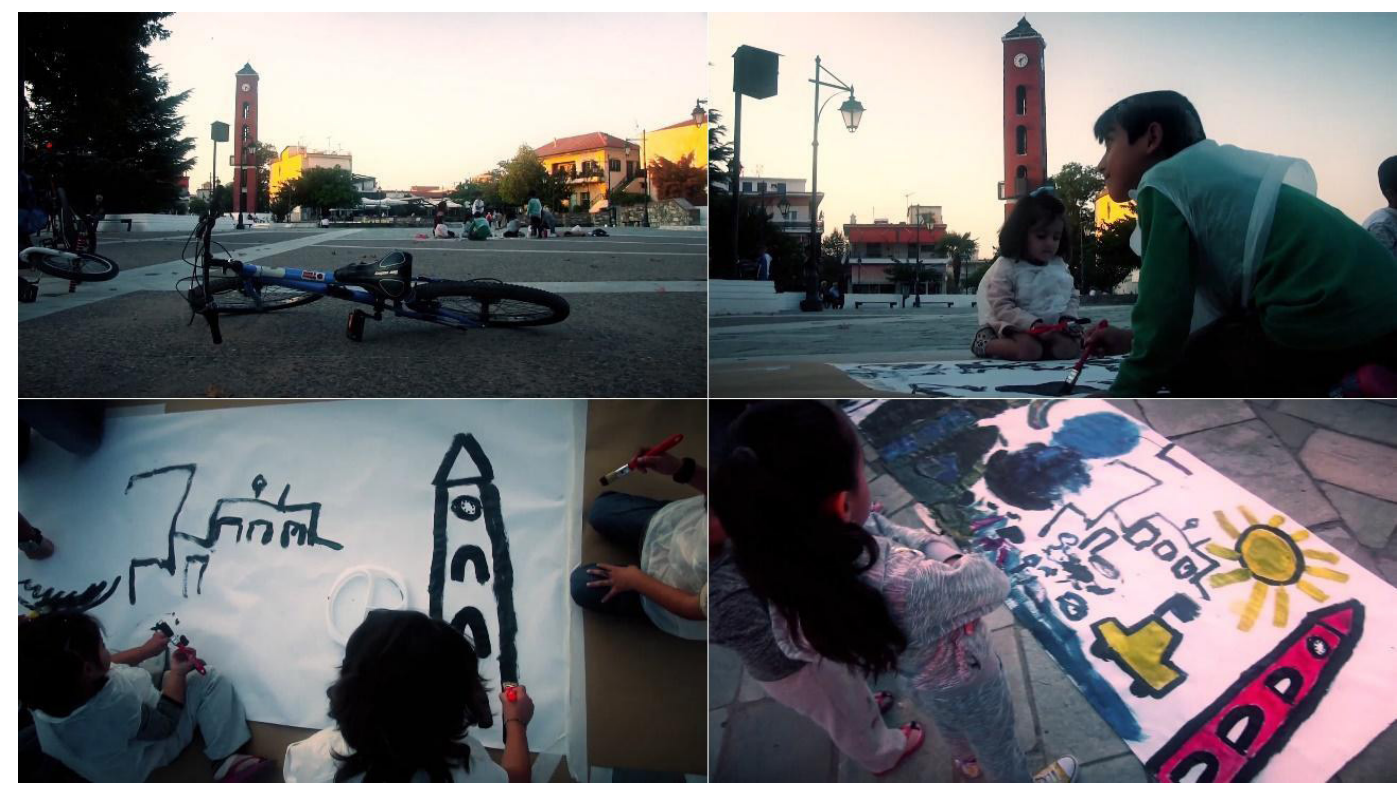

4. Habitar la mirada. En las fases previas se ha realizado una aproximación reflexiva paulatina desde la acción gráfica hasta la pictórica, participando de la interacción entre colectivo e individuo, para finalmente desembocar en la presente fase que pretende un ejercicio metafórico en el que se visibilice de manera poética la acción inclusiva del sujeto en su comunidad. Dentro de las instalaciones de OCC se implementa esta última fase de intervención pedagógica donde se propone una dinámica de generación de espacio virtual interactivo. Para ello se utilizan los recursos pertinentes que permiten generar un pequeño plató de chroma key y realizar un efecto chroma en directo: fondo verde, ordenador con dispositivo de videocámara, pantalla de proyección, proyector y software para generar ese chroma key en tiempo real. De esta manera, utilizando estos recursos y la estrategia para implementar un chroma se tiene la posibilidad de introducir virtualmente a los niños en el espacio que se desee. En este caso, fundamentalmente utilizamos para la creación de ese espacio virtual tanto las pinturas realizadas individualmente como el trabajo colectivo de pintura grupal, realizados los días anteriores, concretamente en la fase 3. Así, con una acción que se nutre de patrones del live cinema y el cine expandido, los niños y niñas se observan dentro de sus propias pinturas, de sus propias creaciones, convirtiéndose en un juego de reimaginación del entorno. Un ejercicio que se vive con un carácter lúdico y festivo donde los niños disfrutan de una experiencia postdigital que potencia los lazos comunitarios e identitarios: poder habitar sus representaciones gráfi- 
cas no solo es un juego, sino un símbolo poético de cómo la mirada que proyectamos pertenece al sujeto $\mathrm{y}$ al grupo social.

Como epílogo de todas las fases resultantes, y como parte del proceso educativo, después de las intervenciones, se realiza días un ejercicio de exhibición pública del documento audiovisual que muestra todo el proceso recorrido: Territorios de la mirada (Territories of the gaze). La exhibición del trabajo para toda la comunidad dentro de las instalaciones de OCC se vive como un acontecimiento festivo, pero igualmente evaluativo, donde el diálogo y valoración entre pares y su comunidad es necesario, donde los protagonistas, los niños y niñas que participan, se reconocen como eje principal del aprendizaje.

Figura 8

Habitar la mirada. Conjunto de fotogramas de Territorios de la mirada (Territories of the gaze) (2019), del colectivo Communiars

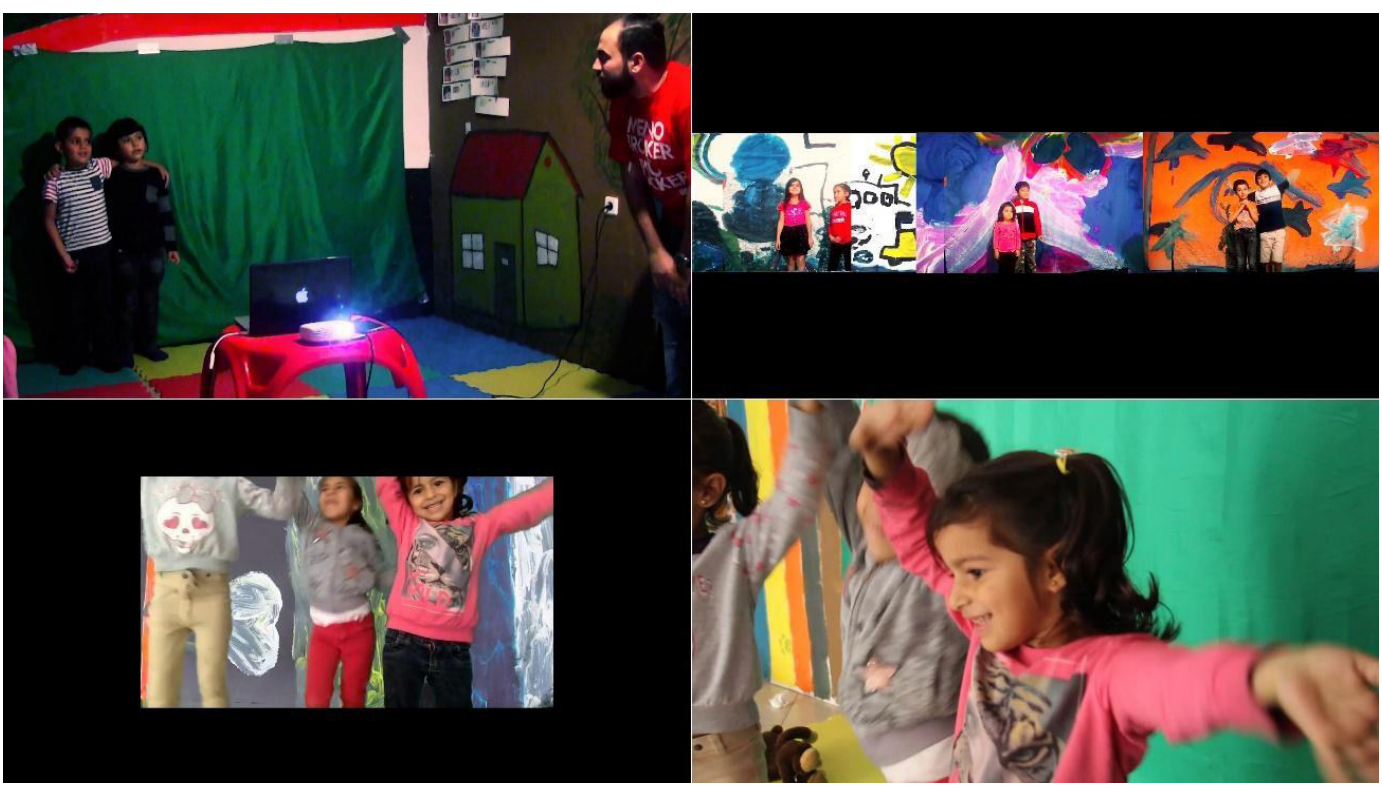

\section{Discusión y conclusiones}

La tradición educativa por la reconstrucción social apunta a que es fundamental ejercer diferentes prácticas para conformar un programa de artes y educación que asuma este enfoque: en primer lugar, ejercer la democracia activamente en un entorno educativo; igualmente, posibilitar a los estudiantes que aprendan a analizar sus propias situaciones; en tercer lugar, potenciar que el alumnado adquiera instrumentos de acción social para llevar a cabo estas acciones anteriores de ejercicio de la democracia y de análisis de situaciones; y por último, fomentar estrategias de alianza y de trabajo colectivo con el objetivo de superar barreras de género, raza, discapacidad y clase social. Propuestas que reorientan la educación hacia un terreno de solidaridad (Escaño, 2020) y crecimiento social, aspectos que se reclaman para el desarrollo óptimo de la educación artística: una educación artística con bases liberadoras (Barbosa, 2019). Sin embargo, esas propuestas basadas en Grant y Sleeter, y recogidas por Arthur Efland, Kerry Freedman y Patricia Sturh (2003) a pesar de mantener una trayectoria educativa de décadas, fueron expuestas en los años 90 , hoy mantienen una vigencia necesaria. En este contexto de ideas hay que destacar que la raíz de una educación orientada hacia la transformación social tiene un recorrido histórico aún más extenso, sus antecedentes contemporáneos nos acercan así a las bases pedagógicas del pensamiento educativo no bancario de Paulo Freire y la pedagogía crítica. Solo hay que señalar el movimiento ecopedagógico más reciente y las incursiones educativas y freirianas que desde la era postdigital revisitan sus propuestas (Jandrić y Ford, 2020). Del mismo modo, se antoja necesario recuperar la noción de Investigación Educativa basada en las Artes, donde la praxis docente y las acciones que desencadena nos conduce a "un espacio de integración de la acción artística como modelo de investigación educativa” (Mesías-Lema, 2021, p. 5). 
En este marco conceptual encaja la propuesta pedagógica Territorios de la mirada. Una propuesta que, a través de estrategias visuales, mantenía el objetivo de ejercer un trabajo inclusivo en relación con la comunidad migrante refugiada de Polykastro, en este caso orientada a los niños y niñas usuarios de OCC. Las prácticas pedagógicas desarrolladas en la Kindergarten de OCC han supuesto un trabajo educativo de interés muy relevante para el proyecto de cooperación puesto en marcha, favoreciendo la atención y aproximación hacia el entorno de la comunidad, potenciando lazos de identidad, apertura cultural e inclusión social. Una acción educativa que podríamos denominar al mismo tiempo artivismo ya que, en definitiva, busca sensibilizar sobre un problema compartido por un colectivo determinado; conectando vidas, necesidades, y dejando espacio para la acción inesperada (Aladro-Vico et al., 2018; Desai, 2017; Mesías-Lema, 2018).

De esta manera, ha servido para evaluar y corroborar diferentes aspectos educativos que debemos tener en cuenta en el futuro proyecto de cooperación a largo plazo. Entre estos aspectos destacamos como conclusiones aquellas relacionadas con los objetivos específicos para con el proyecto global, los cuales estaban orientados hacia la labor de verificación y validación de posibilidades:

La formación del profesorado (voluntariado internacional o residente) que pueda implicarse en un proyecto de educación artística es más factible y viable dentro del campo de la educación infantil. Es decir, la Kindergarten ofrece más estabilidad en comparación con otros proyectos desarrollados por OCC y este hecho nos permite partir de una relativa seguridad a la hora de poder formar en el futuro al voluntariado que pueda participar en ejercicios de educación artística. Con la intervención pedagógica hemos podido comprobar que la participación de la comunidad, tanto los voluntarios internacionales como voluntarios residentes, ha sido completa, fluida y muy activa.

Por otro lado, trabajando con niños de edades tempranas es más asequible salvar diferencias culturales que pudieran dificultar la comunicación. La actitud y compromiso en tareas pedagógicas artísticas con un carácter lúdico y dinámico siempre son bien recibidas por parte del alumnado de cualquier nivel educativo, pero en edades tempranas sus resultados son muy evidentes. Así podemos observarlo en ejemplos que, salvando las distancias culturales, participan igualmente de espacios necesitados de una inclusión social y educativa como los proyectos desarrollados por el colectivo Communiars en India, durante el periodo comprendido entre 2015 y 2018 Colectivo al que el equipo de cooperación pertenece (Escaño, 2019; Escaño et al., 2021).

Finalmente, a partir del desarrollo de esta estrategia pedagógica de Territorios de la mirada podemos concluir que la intervención realizada con niñas y niños de estas edades ha facilitado una mejor imbricación entre la comunidad griega y migrante, aspecto esencial en los objetivos de un proyecto de cooperación de estas características. Esto queda motivado por una mejor inclusión en diferentes niveles y factores, los cuales se corresponden con los criterios de valoración previamente anunciados: en primer lugar, una potenciación de las familias migrantes en la comunidad, donde no solo se benefician los niños y niñas participantes, sino todo su entorno inmediato; asimismo, una mejor inclusión de la comunidad en relación con la institución: los nexos educativos se fortalecen inevitablemente en el momento en que las actividades, como las desarrolladas, se puedan abrir la participación de otros niños autóctonos de la zona; y finalmente, con este tipo de intervenciones en un entorno estable educativo es más asequible la colaboración de universidades internacionales y nacionales.

\section{Agradecimientos}

La presente investigación es parte del proyecto de Cooperación Internacional "Artes, cultura y educación para el desarrollo. Educación y cultura para el empoderamiento personal y social de la comunidad migrante refugiada en Polykastro, Grecia”. Proyecto aprobado en convocatoria competitiva de ayudas para actividades y proyectos de cooperación al desarrollo 2017/2018 de la Universidad de Sevilla.

Mención especial: gracias a la implicación y labor de todos los miembros de Open Cultural Center. Agradecemos de manera especial el compromiso de los miembros de la organización y a su voluntariado residente. 


\section{Referencias}

Agra, M. J. (2005). El vuelo de la mariposa. La investigación artístico-narrativa como herramienta de formación. En R. Marín-Viadel (Coord.), La investigación en educación artística (pp. 127-150). Editorial Universidad de Granada.

Aladro-Vico, E., Jivkova-Semova, D. y Bailey, O. (2018). Artivism: A new educative language for transformative social action. Comunicar, 57, 9-18. https://doi.org/10.3916/C57-2018-01

Anttila, E. y Suominen, A. (2019). Critical articulations of hope from the margins of arts education. International perspectives and practices. Routledge. https://doi.org/10.4324/9781351111195

Association for Supervision and Curriculum Development. (1992). Resolutions 1992. Development, Association for Supervision and Curriculum.

Barbosa, A. M. (2019). A imagem no ensino da arte. Perspectiva.

Carrillo-Jaimes, C. S. y Arcos-González, P. I. (2004). Prioridades de intervención para la fase aguda de emergencias complejas formuladas por nueve agencias de ayuda humanitaria. Revista Española de Salud Pública, 78(6), 679690. https://doi.org/10.1590/S1135-57272004000600003

Chalmers, F. G. (2003). Arte, educación y diversidad cultural. Paidós.

Communiars. (2021). Communiars. http://www.communiars.org

Desai, D. (2017). Artistic activism in dangerous times: Teaching and learning against the grain. Visual Inquiry: Learning \& Teaching Art, 6(2), 135-144. https://doi.org/10.1386/vi.6.2.135_2

DeWalt, K. M. y DeWalt, B. R. (2011). Participant observation. A guide for fieldworkers. Altamira Press.

Efland, A., Freedman, K. y Sturh, P. (2003). Educación en el arte posmoderno. Paidós.

Eisner, E. y Barone, T. (2012). Arts based research. Sage.

Escaño, C. (Coord.). (2019). Lo que se ve no existe. Artes, imagen y educación para el desarrollo. UNED.

Escaño, C. (2020). La solidaridad de lo común frente a las prácticas culturales colonizadoras. Communiars. Revista de Imagen, Artes y Educación Crítica y Social, 3, 6-9.

Escaño, C., Mesías-Lema, J. M. y Mañero, J. (2021). Empowerment of the refugee migrant community through a cooperation project on art education in Greece. Development in Practice. https://doi.org/10.1080/09614524.2021.1944986

Escaño, C., Maeso-Broncano, A. y Mañero, J. (2021). Art education and development cooperation: A project in educational centres for children with special needs in India. International Journal of Art \& Design Education, 4O(2), 404-419. https://doi.org/10.1111/jade.12358

Fondo de las Naciones Unidas para la Infancia. (2017). Education uprooted. For every migrant, refugee and displaced child, education. UNICEF.

Hirsu, L., Arizpe, E. y McAdam, J. E. (2020). Cultural interventions through children's literature and arts-based practices in times of disaster: A case study of reading mediators' response to the Mexican earthquakes. International Journal of Disaster Risk Reduction, 51(3), 1-8. https://doi.org/10.1016/j.ijdrr.2020.101797

Jandrić, P. y Ford, D. R. (2020). Postdigital ecopedagogies: Genealogies, contradictions, and possible futures. Postdigital Science and Education. https://doi.org/10.1007/s42438-020-00207-3

Marín Viadel, R. (2005). La investigación educativa basada en las artes visuales o “arteinvestigación educativa”. En R. Marín Viadel (Coord.), La investigación en educación artística (pp. 223-274). Editorial Universidad de Granada.

Marín Viadel, R. (2011). La investigación en educación artística. Educatio Siglo XXI, 29(1), 211-230.

Massot-Lafon, I., Dorio-Alcaraz, I. y Sabariego-Puig, M. (2009). Estrategias de recogida y análisis de la información. En R. Bisquerra (Ed.), Metodología de la investigación educativa (pp. 329-366). La Muralla.

Mesías-Lema, J. M. (2018). Artivism and social conscience: Transforming teacher training from a sensibility standpoint. Comunicar, 57, 19-28. https://doi.org/10.3916/C57-2018-02 
Mesías-Lema, J. M. (2021). Arts-based educational research no longer needs martyrs: A small tribute to Mark Rothko's teaching practice. IJABER. International Journal of Arts-based Educational Research, 1(1), 1-6. https://doi.org/10.17979/ijaber.2021.1.1.7617

Oficina del Alto Comisionado de las Naciones Unidas para los Refugiados. (2020). Tendencias globales. Desplazamiento forzado en 2019. ACNUR.

OCC. (2020). Memoria de actividades 2019. https://openculturalcenter.org/wp-content/uploads/2020/09/OCC-Spain_Annual-_Report_2019_Spanish.pdf

OCC. (2021). Open cultural center. http://www.openculturalcenter.org

Ortegón, E., Pacheco, J. F. y Prieto, A. (2015). Metodología del marco lógico para la planificación, el seguimiento y la evaluación de proyectos y programas. CEPAL.

Robertson, J. (2014). Dirty teaching. A beginner's guide to learning outdoors. Independent Thinking Press.

Rosenberger, S. (2019). Navigating the representative-politics-liberal-rights dilemma: Social policy designs for nonremoved migrants. Journal of Immigrant \& Refugee Studies, 17(1), 11-26. https://doi.org/10.1080/15562948.2018.1489089

Ruiz-Olabuénaga, J. (1999). Metodología de la investigación cualitativa. Universidad de Deusto.

Sarah, S. y Delvino, N. (2019). Municipal activism on irregular migrants: The framing of inclusive approaches at the local level. Journal of Immigrant \& Refugee, 17(1), 27-43. https://doi.org/10.1080/15562948.2018.1519867

Saura, A. (2015). Arte, educación y justicia social. Opción, 31(6), 765-789.

Sleeter, C. y Grant, C. (1988). An analysis of multicultural education in the U.S. Harvard Educational Review, 57(4), 421-445. https://doi.org/10.17763/haer.57.4.v810xr0v3224x316

Triandafyllidou, A. (2018). A "refugee crisis" unfolding: "Real” events and their interpretation in media and political debates. Journal of Immigrant and Refugee Studies, 16(2), 198-216. https://doi.org/10.1080/15562948.2017.1309089

Wagner, M., Dimitriadi, A., Donnell, R., Kraler, A., Perumadan, J., Schlotzhauer, J. H., Simic, I. y Yabasun, D. (2016). The implementation of the common european asylum system. LIBE.

\section{Breve cv de los/as autores/as}

\section{Carlos Escaño}

Profesor Titular de la Universidad de Sevilla. Doctor en Bellas Artes por la Universidad de Sevilla (1999) y doctor por la Universidad Nacional de Educación a Distancia UNED (2017) en el campo de la educación y la comunicación en entornos digitales. Sus principales intereses docentes e investigadores se centran en la educación artística ubicada en la intersección de las artes y la cultura audiovisual y digital, utilizando un enfoque pedagógico crítico orientado a la transformación social. Es editor jefe de la revista científica Communiars, director del grupo de investigación Educación y Cultura Audiovisual (HUM401) (http:// grupos.us.es/ecav/) y coordinador de proyectos de cooperación internacional como "Educación Artística y Desarrollo Humano” (2015-2018), India, y “Artes, cultura y educación para el desarrollo” (2019), Grecia. Email: jcescano@us.es

ORCID ID: https://orcid.org/0000-0002-8018-2347

\section{Julia Mañero}

Doctora en Educación por la Universidad de Sevilla (2020). Sus intereses de investigación y docencia incluyen la educación artística y la educación digital desde una perspectiva crítica y social. Miembro del grupo de investigación Educación y Cultura Audiovisual (HUM401) (http://grupos.us.es/ecav/), editora asociada de la revista Communiars y colaboradora docente en el Máster de Comunicación y Educación en Red de la Universidad Nacional de Educación a Distancia (UNED). Miembro activo del colectivo Commu- 
niars y del grupo de cooperación Pedagogías Críticas y Educación Artística de la Universidad de Sevilla, habiendo participado en proyectos e investigaciones en países como India y Grecia. Email: juliamanero@ us.es

ORCID ID: https://orcid.org/0000-0003-2721-6947

\section{José María Mesías-Lema}

Profesor Titular de educación artística en la Universidade da Coruña. Ha sido vicedecano de Relaciones Internacionales, vicerrector adjunto y director del Centro Cultural de Arte NORMAL (www.istoenormal. org) en la misma universidad. También dirige el grupo de investigación Artefacto-UDC (www.arte-facto. org), que se centra en los procesos artísticos contemporáneos en diversos contextos educativos. Es educador artivista y promotor de aprendizaje sensible para la defensa de los derechos humanos, con un doctorado en Artes Visuales y Educación por la Universidad de Granada. Sus últimas investigaciones generan proyectos en escuelas, donde los artistas habitantes construyen espacios como laboratorios experimentales en educación artística. Email: jose.mesias@udc.es

ORCID ID: https://orcid.org/0000-0001-8278-7115 